\title{
Evaluation of Weed Control Options for Herbicide Resistant Transgenic Stacked (TC 1507 X NK603) and Conventional Maize Hybrids for Higher Productivity
}

\author{
Sivagamy Kannan*, Chinnusamy Chinnagounder \\ Department of Agronomy, Tamil Nadu Agricultural University, Coimbatore, India. \\ Email: *ksivagamy@yahoo.com
}

Received June $4^{\text {th }}, 2013$; revised July $1^{\text {st }}, 2013$; accepted July $25^{\text {th }}, 2013$

Copyright (C) 2013 Sivagamy Kannan, Chinnusamy Chinnagounder. This is an open access article distributed under the Creative Commons Attribution License, which permits unrestricted use, distribution, and reproduction in any medium, provided the original work is properly cited.

\begin{abstract}
Weeds are posing a serious problem in maize. The congenial climatic conditions encourage more weed growth in the widely spaced crop like maize and cause yield reduction to the tune of 29 to 70 percent. A field experiment was conducted for two years at Tamil Nadu Agricultural University, Coimbatore during kharif seasons of 2010 and 2011 to evaluate the weed management options for transgenic stacked (TC 1507 \& NK 603) and conventional maize hybrids. The experiments were conducted with the following objective to evaluate the weed control efficiency and crop productivity with K salt of glyphosate formulations under field conditions. Treatments consisted of two transgenic stacked hybrids named 30V92 and 30B11 applied with glyphosate as early post emergence at 900 and $1800 \mathrm{~g}$ a.e ha ${ }^{-1}$ during kharif, 2010 and conventional maize hybrids named 30V92 and 30B11 applied with glyphosate by controlled droplet application method at 900, 1350 and $1800 \mathrm{~g}$ a.e $\mathrm{ha}^{-1}$ during kharif, 2011 compared with non transgenic counterpart maize hybrids applied with pre emergence atrazine at $0.5 \mathrm{~kg} \mathrm{ha}^{-1}$ followed by one hand weeding on 40 DAS with and without insect management. Based on the two years field experimentation, it was found that early post emergence application of glyphosate at $1800 \mathrm{~g}$ a.e ha ${ }^{-1}$ gave significantly lower weed density, weed dry weight and higher weed control efficiency at all the intervals. Higher grain yield was registered with post emergence application of glyphosate at $1800 \mathrm{~g}$ a.e $\mathrm{ha}^{-1}$ in transgenic and conventional maize hybrid of 30V92 $\left(12.21 \mathrm{t} \mathrm{ha}^{-1}\right.$ and $\left.11.23 \mathrm{tha}^{-1}\right)$ during both seasons of the study. Unweeded control accounted for grain yield which in turn reflected through higher weed index of 58.39 and 57.26 per cent, respectively during both the years, due to heavy competition of weeds for nutrients, space and light.
\end{abstract}

Keywords: Herbicide Tolerant Maize; Weed Density; Weed Dry Weight; Yield Attributes; Grain Yield

\section{Introduction}

The development of crop cultivar with resistance to selected herbicides has a positive impact on agricultural production. Selection of proper herbicides is essential for successful weed management in all crop production systems [1]. Maize was an ideal crop due to availability of the plant to acclimate to many soil and climate conditions. It is an important cereal crops cultivated worldwide. It is not only an important human nutrient source, but also a basic element of animal feed and raw material for manufacture of many industrial products. The major yield reducing factors for maize cultivation in India are weeds and insects. Weeds cause considerable yield loss due to

\footnotetext{
"Corresponding author.
}

competition of resources with maize crop [2].

Herbicidal weed control seems to be a competitive and promising way to control weeds at initial stages of crop growth. Post emergence herbicides have been achieving adequate weed control programmes, due to its broad spectrum of activity, excellent crop safety, convenience and flexibility [3]. This necessitates the development and testing of selective early post emergence herbicides for weed control in maize. Glyphosate is a foliar applied, broad spectrum, post emergence herbicide capable of controlling annual, perennial grasses and dicotyledonous weeds. The introduction of glyphosate resistant crops has created new opportunities for the use of effective, non selective herbicides like glyphosate as selective weed control in crop production. Prior to the introduction of 
glyphosates resistant crops, glyphosate is being applied to control existing vegetation prior to sowing the crops.

Now, it can be used as post emergence herbicide in glyphosate resistant crops [4]. Herbicide resistant corn plants confer tolerance to glyphosate by production of glyphosate resistant CP4 5-enolpyruvyl shikimate-3 phosphate synthase (CP4 EPSPS) proteins. Transgenic stacked hybrid maize (TC1507 X NK 603) was developed for preventing yield losses for maize crop due to pests and weeds and to improve productivity. Post emergence application of glyphosate at $1800 \mathrm{~g}$ a.e ha ${ }^{-1}$ gave significantly lower weed index, weed dry weight and high weed control efficiency at all the intervals. Post emergence application of glyphosate at $1800 \mathrm{~g}$ a.e ha ${ }^{-1}$ in transgenic maize and post emergence control droplet application method of glyphosate at $1800 \mathrm{~g}^{2}$.e ha ${ }^{-1}$ in conventional maize hybrid (30V92) recorded high productivity and profitability. In view of the above facts, an experiment on "Evaluation of weed management options for transgenic stacked and non transgenic maize hybrids' was formulated with the following objectives:

1) To evaluate the weed management efficiency of glyphosate $\mathrm{K}$ salt formulation under field conditions and carry-over on succeeding crops;

2) To study the effect of different weed management practices on maize growth and development.

\section{Materials and Methods}

\subsection{Experimental Site and Initial Soil Characteristics}

Field experiments were laid out during kharif seasons of 2010 \& 2011 in Eastern block farm of Tamil Nadu Agricultural University, located at Coimbatore, India. The geographical location of the experimental site is situated in western agro climatic zone of Tamil Nadu at $11^{\circ} \mathrm{N}$ longitude and $77^{\circ} \mathrm{E}$ latitude with an altitude of $426.7 \mathrm{~m}$ above MSL and the farm receives the total annual rainfall of $674 \mathrm{~mm}$ in 45.8 rainy days. The soil of the experimental site was sandy clay loam in texture $32.48 \%$ clay, $18.50 \%$ silt and $28.96 \%$ coarse sand) with low available nitrogen,medium in available phosphorous and high in available potassium. The soil analysed 260, 11.90 and $490 \mathrm{Kg} \mathrm{ha}^{-1}$ of $\mathrm{KMnO}_{4}-\mathrm{N}$, Olsen-P and $\mathrm{NH}_{4} \mathrm{OAc}-\mathrm{K}$, respectively with EC of $0.16 \mathrm{dSm}^{-1}$, $\mathrm{Ph}$ of 8.11 and organic carbon of $0.31 \%$.

\subsection{Experimental Design, Selection of Cultivar and Sowing}

The experiment was laid out in randomized complete block design (RBD) with sixteen treatments and replicated thrice. The gross plot size adopted was $(5 \mathrm{~m} \times 3.6$ m) $18 \mathrm{Sq}$ meter. The adopted spacing between the rows and plants were $60 \mathrm{~cm}$ and $25 \mathrm{~cm}$ respectively. Herbicide tolerant transgenic maize test hybrids namely 30V92, 30B11 and conventional hybrids of 30V92, 30B11, BIO 9681 and COHM5 during kharif, 2010. Conventional maize hybrids 30V92, 30B11, BIO 9681 and COHM5 were raised during kharif, 2011. After sowing the seed, immediate light irrigation was given to the crop for uniform germination.

\subsection{Treatment Details}

The experiment I and II comprises of sixteen treatments. The experiment I during kharif, 2010 comprises two transgenic stacked hybrids 30V92 and 30B11 with post emergence of glyphosate at 900 and $1800 \mathrm{~g}$ a.e ha ${ }^{-1}$ and conventional maize hybrids of $30 \mathrm{~V} 92$ with pre emergence atrazine at $0.5 \mathrm{~kg} \mathrm{ha}^{-1}$ on 3 DAS $f b$ HW 40 DAS and insect control, 30V92 no weeding and insect control, $30 \mathrm{~V} 92$ no weeding and no insect control, conventional maize hybrid of 30B11 with pre emergence atrazine at $0.5 \mathrm{~kg} \mathrm{ha}^{-1}$ on 3 DAS $f b$ HW 40 DAS and insect control, 30B11 no weeding and insect control, 30B11 no weeding and no insect control, conventional maize hybrids of BIO 9681 with pre emergence atrazine at $0.5 \mathrm{~kg} \mathrm{ha}^{-1}$ on 3 DAS $f b$ HW 40 DAS and insect control, BIO 9681 no weeding and no insect control, COHM5 with pre emergence atrazine at $0.5 \mathrm{~kg} \mathrm{ha}^{-1}$ on 3 DAS $f b$ HW 40 DAS and insect control, COHM5 no weeding and no insect control.

The experiment II during kharif, 2011 comprises of conventional maize hybrid of $30 \mathrm{~V} 92$ with post emergence controlled droplet application of glyphosate at 900 , 1350 and $1800 \mathrm{~g}$ a.e ha ${ }^{-1}, 30 \mathrm{~B} 11$ hybrid with post emergence glyphosate at 900,1350 and $1800 \mathrm{~g}$ a.e ha ${ }^{-1}, 30 \mathrm{~V} 92$ with pre emergence atrazine at $0.5 \mathrm{~kg} \mathrm{ha}^{-1}$ on 3 DAS $f b$ HW 40 DAS and insect control, 30V92 no weeding and insect control, 30V92 no weeding and no insect control, $30 \mathrm{~B} 11$ with pre emergence atrazine at $0.5 \mathrm{~kg} \mathrm{ha}^{-1}$ on 3 DAS $f b$ HW 40 DAS and insect control, 30B11 no weeding and insect control,30B11 no weeding and no insect control, BIO 9681 with pre emergence atrazine at $0.5 \mathrm{~kg}$ $\mathrm{ha}^{-1}$ on 3 DAS $f b$ HW 40 DAS and insect control, BIO 9681 no weeding and no insect control and COHM5 with pre emergence atrazine at $0.5 \mathrm{~kg} \mathrm{ha}^{-1}$ on 3 DAS $f b \mathrm{HW}$ 40 DAS and insect control, COHM5 no weeding and no insect control.

The herbicides as per the treatments schedule were applied as pre emergence at third day after sowing, glyphosate application at 2 - 4 leaf stage of weeds $(20-25$ DAS of maize). Hand operated knapsack sprayer fitted with a flat fan type nozzle (WFN 40) was used for spraying the herbicides adopting a spray volume of 250 litres 
$\mathrm{ha}^{-1}$. The recommended dose of 150:75:75 Kg of NPK $\mathrm{ha}^{-1}$ in the form of urea, single super phosphate and muriate of potash. During the course of experiment, the data were revealed our predominant weed flora, weed density and dry weight with grain yield.

\subsection{Observation on Weeds}

\subsubsection{Weed Density}

The weed count was recorded species wise using $0.5 \mathrm{~m} \times$ $0.5 \mathrm{~m}$ quadrated four randomly fixed places in each plot and the weeds falling within the frames of the quadrat were counted, recorded and the mean values were expressed in number $\mathrm{m}^{-2}$. The density of grasses, sedges and broadleaved weeds and the total weeds were recorded at 20,40, 60 and 80 days after sowing and expressed in number $\mathrm{m}^{-2}$.

\subsubsection{Weed Dry Weight}

The weeds falling within the frames of the quadrant were collected, categorized into grasses, sedge and broadleaved weeds, shade dried and later dried in hot air oven at $80^{\circ} \mathrm{C}$ for $72 \mathrm{hrs}$. the dry weight of grasses, sedge and broadleaved weeds were recorded separately at 20, 40 and 60 days after sowing and expressed in $\mathrm{kg} \mathrm{ha}^{-1}$.

\subsubsection{Weed Control Efficiency}

Weed control efficiency was calculated as per the procedure [5]

$$
\mathrm{WCE} \%=\frac{\mathrm{WDc}-\mathrm{WD}_{\mathrm{t}}}{\mathrm{WD}_{\mathrm{c}}} \times 100
$$

where,

WCE-Weed control efficiency (per cent);

$\mathrm{WD}_{\mathrm{c}}$-Weed biomass $\left(\mathrm{g} \mathrm{m}^{-2}\right)$ in control plot;

$\mathrm{WD}_{\mathrm{t}}$-Weed biomass $\left(\mathrm{g} \mathrm{m}^{-2}\right)$ in treated plot.

\subsubsection{Weed Index}

Weed index was calculated as per the method [6]

$$
\mathrm{WI}=\frac{\mathrm{X}-\mathrm{Y}}{\mathrm{X}} \times 100
$$

where, $\mathrm{X}=$ Yield $\left(\mathrm{Kg} \mathrm{ha}^{-1}\right)$ from minimum weed competition plot, $\mathrm{Y}=$ yield $\left(\mathrm{Kg} \mathrm{ha}^{-1}\right)$ from the treatment plot for which WI is to be worked out.

\subsection{Statistical Analysis}

The data were statistically analysed following the procedure given by Gomez and Gomez (2010) for randomised block design. The data pertaining to weeds and germination were transformed to square root scale of $\sqrt{(X+2)}$ and analysed as suggested by [7]. Whenever significant difference existed, critical difference was constructed at five per cent probability level.

\section{Results and Discussion}

\subsection{Effect on Weeds}

Weed flora of the experimental field in maize was predominantly consisted of twelve species of broad leaved weeds, five species of grasses and a sedge weed. The dominant broadleaved weeds were Trianthema portulacastrum, Datura stramonium, Cleome gynandra, Digera arvensis, Physalis minima and Corchorus olitorius. The dominant grass weeds were Setaria verticillata and $\mathrm{Cy}$ nodon dactylon. Cyperus rotundus was the only sedge present in the experimental field.

\subsection{Weed Density}

During kharif, 2010 season, lower weed density was achieved under non transgenic maize hybrid BIO 9681 and 30B11 with pre emergence application of atrazine at $0.5 \mathrm{Kg} \mathrm{ha}^{-1}$ followed by hand weeding at 20 DAS. Relatively, a higher density was observed under unweeded checks and transgenic maize before imposing post emergence application of glyphosate. At 40 DAS and 60 DAS, lower weed density observed under transgenic maize hybrid 30V92 with post emergence application of glyphosate at $1800 \mathrm{~g}$ a.e ha ${ }^{-1}$ resulted in effective control of broad leaved weeds, grasses and sedges due to its broad spectrum action [8]. This may due to more impressive control of broadleaved weeds like Trainthema portulacastrum, Datura stramonium, Cleome gynandra and Physallis minima. Foliar application of glyphosate was readily and rapidly translocated throughout the actively growing aerial and under ground portions at active growing stage of broadleaved weeds might have blocked the 5Enolpyruvyl shikimate-3-phosphate synthase enzyme and arrest the amino acid synthesis which led to complete control [9] (Table 1). During kharif, 2011 season, post emergence controlled droplet application of glyphosate at conventional maize hybrid of $30 \mathrm{~V} 92$ at $1800 \mathrm{~g}$ a.e ha ${ }^{-1}$ $\left(1.84 \mathrm{Nos} \mathrm{m}^{-2}\right)$ observed lesser total weed density at 40 DAS. Thus glyphosate effectively controlled a broad spectrum of annual and perennial grasses, sedges and broadleaved weeds could be due to increased translocation of glyphosate inside the plant tissues [10] (Table 2).

\subsection{Weed Dry Weight}

Weed dry weight is the most important parameter to assess the weed competitiveness for the crop growth and productivity. Considerable reduction in weed dry weight was recorded with the application of glyphosate at 1800 
Table 1. Effect of glyphosate application on total weed density and total weed dry weight in herbicide tolerant transgenic maize.

\begin{tabular}{|c|c|c|c|c|c|c|c|c|}
\hline \multirow{3}{*}{ Treatments } & \multicolumn{8}{|c|}{ Kharif, 2010} \\
\hline & \multicolumn{3}{|c|}{ Total weed density (No $\mathrm{m}^{-2}$ ) } & \multicolumn{3}{|c|}{ Total weed dry weight $\left(\mathrm{g} \mathrm{m}^{-2}\right)$} & \multicolumn{2}{|c|}{ WCE (\%) } \\
\hline & 20 DAS & 40 DAS & 60 DAS & 20 DAS & 40 DAS & 60 DAS & 20 DAS & 40 DAS \\
\hline $\begin{array}{l}\mathrm{T}_{1}-30 \mathrm{~V} 92 \mathrm{POE} \text { Glyphosate } \\
\text { (a) } 900 \mathrm{~g} \text { a.e ha }\end{array}$ & $15.43(236.22)$ & $2.78(5.75)$ & $3.4(9.63)$ & $7.61(55.94)$ & $1.88(1.52)$ & $2.35(3.54)$ & 0.00 & 98.56 \\
\hline $\begin{array}{l}\mathrm{T}_{2}-30 \mathrm{~V} 92 \mathrm{POE} \text { Glyphosate } \\
\text { (a) } 1800 \mathrm{~g} \mathrm{a} \cdot \mathrm{ha}^{-1}\end{array}$ & $15.33(233.08)$ & $2.04(2.15)$ & $2.35(3.52)$ & $7.37(52.37)$ & $1.58(0.49)$ & $1.87(1.50)$ & 0.00 & 99.53 \\
\hline $\begin{array}{l}\mathrm{T}_{3}-30 \mathrm{~V} 92 \text { POE Glyphosate } \\
\text { (Weedy check) }\end{array}$ & $15.74(245.60)$ & $14.3(202.93)$ & $13.81(188.75)$ & $7.66(56.62)$ & $10.39(106.03)$ & $10.22(102.43)$ & 0.00 & 0.00 \\
\hline $\begin{array}{l}\mathrm{T}_{4}-30 \mathrm{~B} 11 \text { POE Glyphosate } \\
\text { (a) } 900 \mathrm{~g} \text { a.e ha }\end{array}$ & $15.78(246.89)$ & $3.31(8.98)$ & $3.84(12.74)$ & $7.40(52.79)$ & $2.16(2.68)$ & $2.60(4.75)$ & 0.00 & 97.72 \\
\hline $\begin{array}{l}\mathrm{T}_{5}-30 \mathrm{~B} 11 \mathrm{POE} \text { Glyphosate } \\
\text { @ } 1800 \mathrm{~g} \text { a.e ha }\end{array}$ & $16.06(256.07)$ & $2.55(4.50)$ & $3.06(7.35)$ & $7.71(57.41)$ & $1.79(1.20)$ & $2.21(2.87)$ & 0.00 & 98.97 \\
\hline $\begin{array}{l}\mathrm{T}_{6}-30 \mathrm{~B} 11 \text { POE Glyphosate } \\
\text { (Weedy check) }\end{array}$ & $15.81(248.10)$ & $14.5(209.43)$ & $14.42(205.99)$ & $8.18(64.92)$ & $10.94(117.59)$ & $10.22(102.51)$ & 0.00 & 0.00 \\
\hline $\begin{array}{l}\mathrm{T}_{7}-30 \mathrm{~V} 92 \mathrm{PE} \text { atrazine } 0.5 \\
\mathrm{~kg} \mathrm{ha}^{-1}+\mathrm{HW}+\text { Insect Control }\end{array}$ & $7.99(61.85)$ & $7.81(59.00)$ & $5.79(31.48)$ & $3.68(11.57)$ & $5.78(31.43)$ & $4.52(18.39)$ & 80.28 & 72.57 \\
\hline $\begin{array}{l}\mathrm{T}_{8}-30 \mathrm{~V} 92 \text { No Weed control } \\
\text { and only Insect Control }\end{array}$ & $15.45(236.55)$ & 13.64 (183.99) & $12.7(160.36)$ & $7.08(48.17)$ & 9.99 (97.79) & 9.42 (86.77) & 0.00 & 14.66 \\
\hline $\begin{array}{l}\mathrm{T}_{9}-30 \mathrm{~V} 92 \text { No Weed control } \\
\text { and no Insect Control }\end{array}$ & $16.05(255.75)$ & $14.37(204.37)$ & $14.38(204.69)$ & $7.79(58.70)$ & $10.80(114.59)$ & $10.58(109.99)$ & 0.00 & 0.00 \\
\hline $\begin{array}{l}\mathrm{T}_{10}-30 \mathrm{~B} 11 \mathrm{PE} \text { atrazine } 0.5 \\
\mathrm{~kg} \mathrm{ha}^{-1}+\mathrm{HW}+\text { insect control }\end{array}$ & $7.55(55.00)$ & $8.14(64.34)$ & $5.87(32.43)$ & $3.88(13.04)$ & $6.12(35.48)$ & $4.72(20.32)$ & 79.66 & 70.33 \\
\hline $\begin{array}{l}\mathrm{T}_{11}-30 \mathrm{~B} 11 \text { No Weed control } \\
\text { and only Insect Control }\end{array}$ & $15.51(238.44)$ & $13.5(182.38)$ & $13.12(170.11)$ & $7.41(52.92)$ & $10.3(105.35)$ & 9.97 (97.47) & 0.00 & 11.92 \\
\hline $\begin{array}{l}\mathrm{T}_{12}-30 \mathrm{~B} 11 \text { No Weed control } \\
\text { and no Insect Control }\end{array}$ & $16.25(262.00)$ & $15.05(224.47)$ & $15.0(224.57)$ & $8.13(64.14)$ & $11.03(119.61)$ & $10.98(118.66)$ & 0.00 & 0.00 \\
\hline $\begin{array}{l}\mathrm{T}_{13}-\mathrm{BIO} 9681 \mathrm{PE} \text { atrazine } 0.5 \\
\mathrm{~kg} \mathrm{ha}^{-1}+\mathrm{HW}+\text { Insect Control }\end{array}$ & $7.15(49.14)$ & $7.52(54.58)$ & $5.96(33.49)$ & $3.74(11.98)$ & $6.23(36.77)$ & $5.12(24.24)$ & 77.27 & 68.73 \\
\hline $\begin{array}{l}\mathrm{T}_{14}-\mathrm{BIO} 9681 \text { No Weed } \\
\text { control and no Insect Control }\end{array}$ & $14.69(213.70)$ & $13.8(189.93)$ & $14.52(208.94)$ & $7.40(52.71)$ & $10.94(117.62)$ & $10.90(116.82)$ & 0.00 & 0.00 \\
\hline $\begin{array}{l}\mathrm{T}_{15} \text { - CoHM5 PE atrazine } 0.5 \\
\mathrm{~kg} \mathrm{ha}^{-1}+\mathrm{HW}+\text { Insect Control }\end{array}$ & $7.83(59.37)$ & $8.32(67.3)$ & $6.20(36.44)$ & $4.08(14.61)$ & $6.75(43.55)$ & $5.69(30.32)$ & 79.28 & 68.56 \\
\hline $\begin{array}{l}\mathrm{T}_{16}-\text { CoHM5 No Weed control } \\
\text { and no Insect Control }\end{array}$ & $16.38(266.19)$ & $15.24(230.37)$ & $15.79(247.44)$ & $8.52(70.54)$ & $11.8(138.52)$ & $12.16(145.78)$ & 0.00 & 0.00 \\
\hline SEd & 1.34 & 1.11 & 1.06 & 0.65 & 0.83 & 0.80 & - & - \\
\hline $\mathrm{CD}(\mathrm{P}=0.05)$ & 2.74 & 2.27 & 2.17 & 1.34 & 1.70 & 1.63 & - & - \\
\hline
\end{tabular}

Figures in parenthesis are original values must need for differentiating the original and transformed values, DAS—Days After sowing, POE—Post-emergence, PE-Pre emergence, WCE-Weed control efficiency, HW-Hand Weeding. 
Table 2. Effect of glyphosate application on total weed density and total weed dry weight in conventional maize hybrids.

\begin{tabular}{|c|c|c|c|c|c|c|c|c|}
\hline \multirow{3}{*}{ Treatments } & \multicolumn{8}{|c|}{ Kharif, 2011} \\
\hline & \multicolumn{3}{|c|}{ Total weed density $\left(\mathrm{No} \mathrm{m}^{-2}\right)$} & \multicolumn{3}{|c|}{ Total weed dry weight $\left(\mathrm{g} \mathrm{m}^{-2}\right)$} & \multicolumn{2}{|c|}{ WCE (\%) } \\
\hline & 20 DAS & 40 DAS & 60 DAS & 20 DAS & 40 DAS & 60 DAS & $\begin{array}{c}20 \\
\text { DAS }\end{array}$ & $\begin{array}{c}40 \\
\text { DAS }\end{array}$ \\
\hline $\begin{array}{l}\mathrm{T}_{1}-30 \mathrm{~V} 92 \text { POE Glyphosate } \\
\text { (a) } 900 \mathrm{~g} \mathrm{a} . \mathrm{e} \mathrm{ha}^{-1}\end{array}$ & $15.43(236.22)$ & $2.78(5.75)$ & $3.4(9.63)$ & $7.61(55.94)$ & $1.88(1.52)$ & $2.35(3.54)$ & 5.14 & 96.15 \\
\hline $\begin{array}{l}\mathrm{T}_{2}-30 \mathrm{~V} 92 \text { POE Glyphosate } \\
\text { @ } 1350 \mathrm{~g} \mathrm{a}^{\mathrm{e}} \mathrm{ha}^{-1}\end{array}$ & $15.33(233.08)$ & $2.04(2.15)$ & $2.35(3.52)$ & $7.37(52.37)$ & $1.58(0.49)$ & $1.87(1.50)$ & 14.29 & 97.66 \\
\hline $\begin{array}{l}\mathrm{T}_{3}-30 \mathrm{~V} 92 \text { POE Glyphosate } \\
\text { (a) } 1800 \mathrm{~g} \mathrm{a} . \mathrm{e} \mathrm{ha}^{-1}\end{array}$ & $15.74(245.60)$ & $14.3(202.93)$ & $13.81(188.75)$ & $7.66(56.62)$ & $10.39(106.03)$ & $10.22(102.43)$ & 8.73 & 99.14 \\
\hline $\begin{array}{l}\mathrm{T}_{4}-30 \mathrm{~B} 11 \text { POE Glyphosate } \\
\text { (a) } 900 \mathrm{~g} \text { a.e ha }\end{array}$ & $15.78(246.89)$ & $3.31(8.98)$ & $3.84(12.74)$ & $7.40(52.79)$ & $2.16(2.68)$ & $2.60(4.75)$ & 21.41 & 95.86 \\
\hline $\begin{array}{l}\mathrm{T}_{5}-30 \mathrm{~B} 11 \mathrm{POE} \text { Glyphosate } \\
\text { (a) } 1350 \mathrm{~g} \text { a.e ha }\end{array}$ & $16.06(256.07)$ & $2.55(4.50)$ & $3.06(7.35)$ & $7.71(57.41)$ & $1.79(1.20)$ & $2.21(2.87)$ & 14.16 & 97.17 \\
\hline 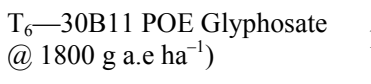 & $15.81(248.10)$ & $14.5(209.43)$ & $14.42(205.99)$ & $8.18(64.92)$ & $10.94(117.59)$ & $10.22(102.51)$ & 11.15 & 98.87 \\
\hline $\begin{array}{l}\mathrm{T}_{7}-30 \mathrm{~V} 92 \mathrm{PE} \text { atrazine } 0.5 \\
\mathrm{~kg} \mathrm{ha}^{-1}+\mathrm{HW}+\text { Insect Control }\end{array}$ & $7.99(61.85)$ & $7.81(59.00)$ & $5.79(31.48)$ & $3.68(11.57)$ & $5.78(31.43)$ & $4.52(18.39)$ & 82.26 & 68.96 \\
\hline $\begin{array}{l}\mathrm{T}_{8}-30 \mathrm{~V} 92 \mathrm{No} \text { Weed control } \\
\text { and only Insect Control }\end{array}$ & $15.45(236.55)$ & $13.64(183.99)$ & $12.7(160.36)$ & $7.08(48.17)$ & $9.99(97.79)$ & $9.42(86.77)$ & 13.97 & 10.25 \\
\hline $\begin{array}{l}\mathrm{T}_{9}-30 \mathrm{~V} 92 \mathrm{No} \text { Weed control } \\
\text { and no Insect Control }\end{array}$ & $16.05(255.75)$ & $14.37(204.37)$ & $14.38(204.69)$ & $7.79(58.70)$ & $10.80(114.59)$ & $10.58(109.99)$ & 0.00 & 0.00 \\
\hline $\begin{array}{l}\mathrm{T}_{10}-30 \mathrm{~B} 11 \mathrm{PE} \text { atrazine } 0.5 \\
\mathrm{~kg} \mathrm{ha}^{-1}+\mathrm{HW}+\text { insect control }\end{array}$ & $7.55(55.00)$ & $8.14(64.34)$ & $5.87(32.43)$ & $3.88(13.04)$ & $6.12(35.48)$ & $4.72(20.32)$ & 80.03 & 65.71 \\
\hline $\begin{array}{l}\mathrm{T}_{11}-30 \mathrm{~B} 11 \mathrm{No} \text { Weed control } \\
\text { and only Insect Control }\end{array}$ & $15.51(238.44)$ & $13.5(182.38)$ & $13.12(170.11)$ & $7.41(52.92)$ & $10.3(105.35)$ & $9.97(97.47)$ & 13.57 & 8.31 \\
\hline $\begin{array}{l}\mathrm{T}_{12}-30 \mathrm{~B} 11 \mathrm{No} \text { Weed control } \\
\text { and no Insect Control }\end{array}$ & $16.25(262.00)$ & $15.05(224.47)$ & $15.0(224.57)$ & $8.13(64.14)$ & $11.03(119.61)$ & $10.98(118.66)$ & 0.00 & 0.00 \\
\hline $\begin{array}{l}\mathrm{T}_{13}-\mathrm{BIO} 9681 \mathrm{PE} \text { atrazine } 0.5 \\
\mathrm{~kg} \mathrm{ha}^{-1}+\mathrm{HW}+\text { Insect Control }\end{array}$ & $7.15(49.14)$ & $7.52(54.58)$ & $5.96(33.49)$ & 3.74 (11.98) & $6.23(36.77)$ & $5.12(24.24)$ & 78.97 & 63.82 \\
\hline $\begin{array}{l}\mathrm{T}_{14}-\mathrm{BIO} 9681 \mathrm{No} \text { Weed control } \\
\text { and no Insect Control }\end{array}$ & $14.69(213.70)$ & $13.8(189.93)$ & $14.52(208.94)$ & $7.40(52.71)$ & $10.94(117.62)$ & $10.90(116.82)$ & 0.00 & 0.00 \\
\hline $\begin{array}{l}\mathrm{T}_{15} \text { - CoHM5 PE atrazine } 0.5 \\
\mathrm{~kg} \mathrm{ha}^{-1}+\mathrm{HW}+\text { Insect Control }\end{array}$ & $7.83(59.37)$ & $8.32(67.3)$ & $6.20(36.44)$ & 4.08 (14.61) & $6.75(43.55)$ & $5.69(30.32)$ & 73.19 & 61.68 \\
\hline $\begin{array}{l}\mathrm{T}_{16}-\mathrm{CoHM} 5 \text { No Weed control } \\
\text { and no Insect Control }\end{array}$ & $16.38(266.19)$ & $15.24(230.37)$ & $15.79(247.44)$ & ) $8.52(70.54)$ & $11.8(138.52)$ & $12.16(145.78)$ & 0.00 & 0.00 \\
\hline SEd & 1.34 & 1.11 & 1.06 & 0.65 & 0.83 & 0.80 & - & - \\
\hline $\mathrm{CD}(\mathrm{P}=0.05)$ & 2.74 & 2.27 & 2.17 & 1.34 & 1.70 & 1.63 & - & - \\
\hline
\end{tabular}

Figures in parenthesis are original values must need for differentiating the original and transformed values, DAS—Days After sowing, POE—Post-emergence, PE-Pre emergence, WCE-Weed control efficiency, HW-Hand Weeding. 
$\mathrm{g}$ a.e ha ${ }^{-1}$ in transgenic $30 \mathrm{~V} 92$ and post emergence controlled application of glyphosate at $1800 \mathrm{~g}$ a.e $\mathrm{ha}^{-1}$ in conventional maize hybrid of 30V92 (1.58 and $1.82 \mathrm{~g}$ $\mathrm{m}^{-2}$ ) at 40 DAS during kharif, 2010 and kharif, 2011 (Tables 1 and 2). This might be weed control as achieved by glyphosate [11] this findings were in accordance with earlier reports that post emergence application followed by pre emergence herbicide reduced total weed dry weight by at least 97 per cent when compared to without glyphosate applied plots [12].

\subsection{Weed Control Efficiency}

Weed control efficiency which indicates the comparative magnitude of reduction in weed dry matter was highly influenced by different weed control treatments. Pre emergence application of atrazine at $0.5 \mathrm{Kg} \mathrm{ha}^{-1}$ followed by hand weeding recorded higher weed control efficiency of 80.28 percent in non transgenic maize hybrid $30 \mathrm{~V} 92$ at 20 DAS. Whereas at 40 DAS after spraying of herbicide, higher weed control efficiency of 99.53 per- cent was recorded in glyphosate at $1800 \mathrm{~g}$ a.e ha ${ }^{-1}$ followed by 30B11was observed 98.97 percent during kharif, 2010 (Table 1). Whereas during kharif, 2011 higher weed control efficiency was observed with glyphosate at $1800 \mathrm{~g}$ a.e $\mathrm{ha}^{-1}$ in conventional maize hybrid of 30V92 registered maximum weed control efficiency of 99.14 percent owing to the fact that registered lesser weed density and weed dry weight (Table 2).

\subsection{Effect on Crop}

During both the years of study, among the weed control treatments, post emergence application of glyphosate at $1800 \mathrm{~g}$ a.e ha ${ }^{-1}$ in transgenic corn hybrid recorded higher grain yield of $12.21 \mathrm{t} \mathrm{ha}^{-1}$ this was 36.64 percent higher than the unweeded check plot of transgenic 30V92 during kharif, 2010, whereas, during kharif, 2011post emergence controlled droplet application of glyphosate at $1800 \mathrm{~g}$ a.e $\mathrm{ha}^{-1}$ in conventional maize hybrid of 30V92 resulted in higher grain yield of $11.23 \mathrm{tha}^{-1}$ (Table 3 and 4). This was 44.79 percent higher than the unweeded check

Table 3. Effect of weed control methods on yield attributes and grain yield in herbicide tolerant transgenic maize.

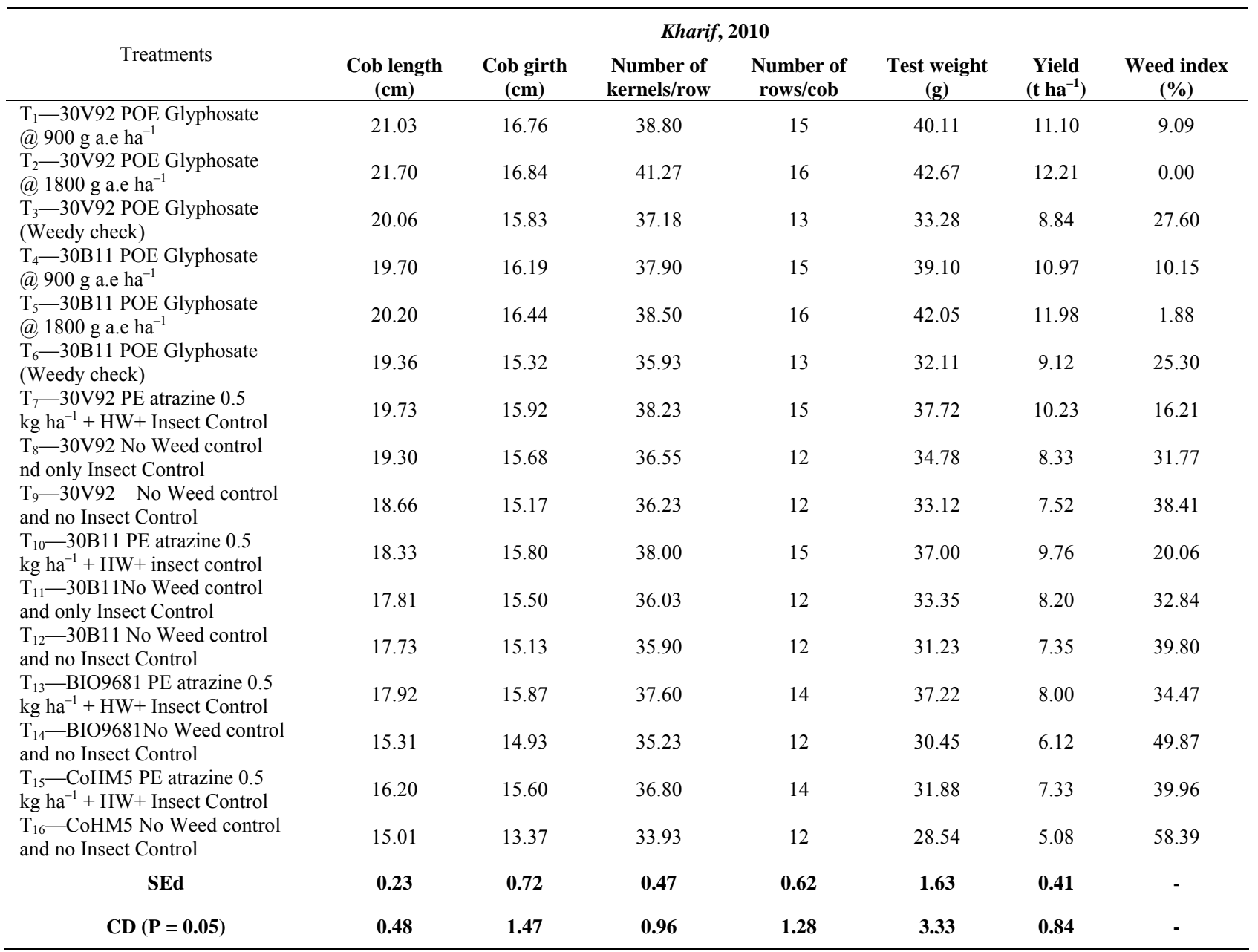


Table 4. Effect of weed control methods on yield attributes and grain yield in conventional maize hybrids.

\begin{tabular}{|c|c|c|c|c|c|c|c|}
\hline \multirow[b]{2}{*}{ Treatments } & \multicolumn{7}{|c|}{ Kharif, 2011} \\
\hline & $\begin{array}{c}\text { Cob length } \\
\text { (cm) }\end{array}$ & $\begin{array}{c}\text { Cob girth } \\
(\mathrm{cm})\end{array}$ & $\begin{array}{l}\text { Number of } \\
\text { kernels/row }\end{array}$ & $\begin{array}{l}\text { Number of } \\
\text { rows/cob }\end{array}$ & $\begin{array}{c}\text { Test weight } \\
\text { (g) }\end{array}$ & $\begin{array}{c}\text { Yield } \\
\left(\mathrm{t} \mathrm{ha}^{-1}\right)\end{array}$ & $\begin{array}{c}\text { Weed index } \\
(\%)\end{array}$ \\
\hline $\begin{array}{l}\mathrm{T}_{1}-30 \mathrm{~V} 92 \text { POE Glyphosate } \\
\text { (a) } 900 \mathrm{~g}^{\mathrm{e}} \mathrm{e} \mathrm{ha}^{-1}\end{array}$ & 18.88 & 15.67 & 38 & 15 & 36.70 & 9.12 & 18.79 \\
\hline $\begin{array}{l}\mathrm{T}_{2}-30 \mathrm{~V} 92 \text { POE Glyphosate } \\
\text { (a) } 1350 \mathrm{~g} \mathrm{a.e} \mathrm{ha}^{-1}\end{array}$ & 19.90 & 16.10 & 38 & 16 & 38.70 & 10.36 & 7.75 \\
\hline $\begin{array}{l}\mathrm{T}_{3}-30 \mathrm{~V} 92 \text { POE Glyphosate } \\
\text { (a) } 1800 \mathrm{~g} \text { a.e ha }\end{array}$ & 21.43 & 16.73 & 41 & 16 & 42.87 & 11.23 & 0.00 \\
\hline $\begin{array}{l}\mathrm{T}_{4}-30 \mathrm{~B} 11 \text { POE Glyphosate } \\
\text { (a) } 900 \mathrm{~g}^{\mathrm{a}} \mathrm{e} \mathrm{ha}^{-1}\end{array}$ & 18.56 & 15.23 & 36 & 14 & 35.52 & 8.25 & 26.54 \\
\hline $\begin{array}{l}\mathrm{T}_{2}-30 \mathrm{~V} 92 \text { POE Glyphosate } \\
\text { (a) } 1350 \mathrm{~g}^{\mathrm{a}} \mathrm{e} \mathrm{ha}^{-1}\end{array}$ & 19.62 & 15.89 & 37 & 15 & 38.10 & 9.52 & 15.23 \\
\hline $\begin{array}{l}\mathrm{T}_{3}-30 \mathrm{~V} 92 \text { POE Glyphosate } \\
\text { (a) } 1800 \mathrm{~g} \text {.e ha }\end{array}$ & 20.12 & 16.33 & 39 & 15 & 40.45 & 10.39 & 7.48 \\
\hline $\begin{array}{l}\mathrm{T}_{7}-30 \mathrm{~V} 92 \mathrm{PE} \text { atrazine } 0.5 \\
\mathrm{~kg} \mathrm{ha}^{-1}+\mathrm{HW}+\text { Insect Control }\end{array}$ & 18.43 & 15.46 & 39 & 14 & 36.55 & 8.72 & 22.35 \\
\hline $\begin{array}{l}\mathrm{T}_{8}-30 \mathrm{~V} 92 \mathrm{No} \text { Weed control } \\
\text { and only Insect Control }\end{array}$ & 16.76 & 14.10 & 33 & 12 & 34.62 & 7.40 & 34.11 \\
\hline $\begin{array}{l}\mathrm{T}_{9}-30 \mathrm{~V} 92 \mathrm{No} \text { Weed control } \\
\text { and no Insect Control }\end{array}$ & 15.79 & 13.96 & 34 & 12 & 34.00 & 6.20 & 44.79 \\
\hline $\begin{array}{l}\mathrm{T}_{10}-30 \mathrm{~B} 11 \mathrm{PE} \text { atrazine } 0.5 \\
\mathrm{~kg} \mathrm{ha}^{-1}+\mathrm{HW}+\text { insect control }\end{array}$ & 18.08 & 15.12 & 36 & 14 & 36.12 & 8.01 & 28.67 \\
\hline $\begin{array}{l}\mathrm{T}_{11}-30 \mathrm{~B} 11 \mathrm{No} \text { Weed control } \\
\text { and only Insect Control }\end{array}$ & 16.12 & 14.65 & 33 & 13 & 34.78 & 6.80 & 39.45 \\
\hline $\begin{array}{l}\mathrm{T}_{12}-30 \mathrm{~B} 11 \text { No Weed control } \\
\text { and no Insect Control }\end{array}$ & 16.01 & 14.23 & 32 & 12 & 33.24 & 6.22 & 44.61 \\
\hline $\begin{array}{l}\mathrm{T}_{13}-\mathrm{BIO} 9681 \mathrm{PE} \text { atrazine } 0.5 \\
\mathrm{~kg} \mathrm{ha}^{-1}+\mathrm{HW}+\text { Insect Control }\end{array}$ & 17.00 & 15.00 & 36 & 14 & 35.41 & 7.10 & 36.78 \\
\hline $\begin{array}{l}\mathrm{T}_{14}-\mathrm{BIO} 9681 \mathrm{No} \text { Weed control } \\
\text { and no Insect Control }\end{array}$ & 15.50 & 13.76 & 32 & 12 & 31.13 & 5.60 & 50.13 \\
\hline $\begin{array}{l}\mathrm{T}_{15}-\text { CoHM5 PE atrazine } 0.5 \\
\mathrm{~kg} \mathrm{ha}^{-1}+\mathrm{HW}+\text { Insect Control }\end{array}$ & 16.10 & 14.17 & 34 & 13 & 34.52 & 6.10 & 45.68 \\
\hline $\begin{array}{l}\mathrm{T}_{16}-\mathrm{CoHM} 5 \mathrm{No} \text { Weed control } \\
\text { and no Insect Control }\end{array}$ & 14.92 & 13.09 & 29 & 12 & 29.23 & 4.80 & 57.26 \\
\hline SEd & 1.12 & 0.67 & 2.49 & 1.35 & 1.65 & 0.80 & - \\
\hline $\mathrm{CD}(\mathrm{P}=\mathbf{0 . 0 5})$ & 2.29 & 1.48 & 5.10 & 2.76 & 3.38 & 1.64 & - \\
\hline
\end{tabular}

plot of conventional maize hybrid. This could be achieved control of weeds with non selective, translocated herbicide, provided the favourable crop growth environment at the establishment stage of the crop itself by minimizing the perennial and annual weeds and increased the seed and stalk yields [13]. This might be due to the fact that the perennial weeds like Cyperus rotundus, Cynodon dactylon, troublesome broadleaved weeds like Trianthema portulacastrum weeds were effectively controlled and might increase the maize yield may be due to better light utilization of narrow row zone and faster canopy closure [14].

Higher yield attributes of increased cob length, cob girth and number of grains per cob and test weight were recorded with post emergence application of glyphosate at $1800 \mathrm{~g}$ a.e ha ${ }^{-1}$ in both the transgenic and conventional maize hybrids of $30 \mathrm{~V} 92$ and 30B11 respectively. This might due to better control of weeds at critical stages and shifted the balance in favour of crop in the utilization of nutrients, moisture, light and space and creates the fa- vourable environment for recording higher growth of maize leading to enhanced yield attributes [15]. Among the weed control treatments methods, lower weed index was recorded with 9.09 and 10.15 per cent in transgenic 30V92 $\left(\mathrm{T}_{1}\right)$ and 30B11 $\left(\mathrm{T}_{5}\right)$ whereas at conventional maize hybrids observed glyphosate at $1350 \mathrm{~g}$ a.e $\mathrm{ha}^{-1}$ recorded lower weed index of 7.75 and 15.23 per cent in non transgenic maize hybrids of 30V92 $\left(\mathrm{T}_{2}\right)$ and 30B11 $\left(\mathrm{T}_{5}\right)$. Unweeded check plots resulted in higher weed index and performed poorly during both the years of study.

\section{Conclusion}

From the results of the field experiments, it could be concluded that post emergence spraying of potassium salt of glyphosate at $1800 \mathrm{~g}$ a.e ha ${ }^{-1}$ in transgenic and conventional maize hybrid of 30V92 enhanced complete control of broad spectrum weeds, could keep the weed density, dry weight reasonable at lower level and enhance higher productvity and profitability with higher grain yield during both the kharif seasons. 


\section{REFERENCES}

[1] J. W. Wilcut, H. D. Coble, A. C. York and D. W. Monks, "The Niche for Herbicide-Resistant Crops in US Agriculture," In: S. O. Duke, Ed., Herbicide-Resistant Crops: Agricultural, Environmental, Economic, Regulatory, and Technical Aspects, CRC Press, Boca Raton, 1996, pp. 213-230

[2] A. Malviya and B. Singh, "Weed Dynamics, Productivity and Economics of Maize (Zea mays) as Affected by Integrated Weed Management under Rainfed Condition," Indian Journal of Agronomy, Vol. 52, No. 4, 2007, pp. 321324.

[3] J. A. Ferrell and W. W. Witt, "Comparison of Glyphosate with Other Herbicides for Weed Control in Corn (Zea mays): Efficacy and Economics," Weed Technology, Vol. 16, No. 3, 2002, pp. 701-706. doi:10.1614/0890-037X(2002)016[0701:COGWOH]2.0. $\mathrm{CO} ; 2$

[4] J. K. Norsworthy, N. R. Burgos and L. R. Oliver, "Differences in Weed Tolerance to Glyphosate Involve Different Mechanism," Weed Technology, Vol. 15, No. 4, 2001, pp. 725-731.

doi:10.1614/0890-037X(2001)015[0725:DIWTTG]2.0.C $\mathrm{O} ; 2$

[5] C. L. Main, A. J. Michael and E. C. Murdock, "Weed Response and Tolerance of Enhanced Glyphosate-Resistant Cotton to Glyphosate," Journal of Cotton Science, Vol. 11, No. 2, 2007, pp. 104-109.

[6] G. S. Gill and K. Vijaya Kumar, "Weed Index-A New Method of Reporting Weed Control Trials," Indian Journal of Agronomy, Vol. 14, No. 2, 1969, pp. 96-98.

[7] G. W. Snedecor and W. G. Cochran, "Statistical Methods," Oxford and IBH Publ. Co., New Delhi, 1967, p. 593.

[8] C. H. Koger and R. N. Reddy, "Glyphosate Efficacy, Ab- sorption and Translocation in Pitted Moringglory (Ipomoea lacunose)," Weed Science, Vol. 53, No. 3, 2005, pp. 277-283. doi:10.1614/WS-04-098R

[9] R. D. Summons, K. J. Grugs, K. S. Andersen, K. A. Johnson and J. A. Sikors, "Re-Evaluating Glyphosate as a Transition-State Inhibitor of EPSP Synthase: Identification of an EPSP Synthase-EPSP Glyphosate Ternary Complex," Biochemistry, Vol. 34, 1995, pp. 6433-6440. doi:10.1021/bi00019a024

[10] U. Suwunnamek and C. Parker, "Control of Cyperus rotundus with Glyphosate, the Influence of Ammonium Sulfate and Other Additives," Weed Research, Vol. 15, No. 1, 1975, pp. 13-19. doi:10.1111/j.1365-3180.1975.tb01090.x

[11] Hafeezullah, "Effect of Different Sowing and Weed Control Methods on Performance of Sunflower," M.Sc. Thesis, Department of Agronomy, Agricultural University of Peshwar, 2000.

[12] A. Kumar and K. S. Thakur, "Influence of Intercropping and Weed Control Measures on Weeds and Productivity of Rainfed Maize (Zea mays)," Indian Journal of Weed Science, Vol. 37, No. 1, 2005, pp. 65-67.

[13] B. E. Tharp, O. Schabenberger and J. J. Kells, "Response of Annual Weed Species to Glufosinate and Glyphosate," Weed Technology, Vol. 13, No. 3, 1999, pp. 542-547.

[14] S. D. Murphy, Y. Yakuba, S. F. Weise and C. J. Swanton, "Effect of Planting Patterns on Inter Row and Competition between Corn and Late Emerging Weeds," Weed Science, Vol. 44, 1996, pp. 865-870.

[15] W. J. Grichar, B. A. Besler, K. D. Brewer and B. W. Minton, "Using Soil-Applied Herbicides in Combination with Glyphosate in a Glyphosate-Resistant Cotton Herbicide Program," Crop Protection, Vol. 23, No. 10, 2004, pp. 1007-1010. doi:10.1016/j.cropro.2004.03.004 